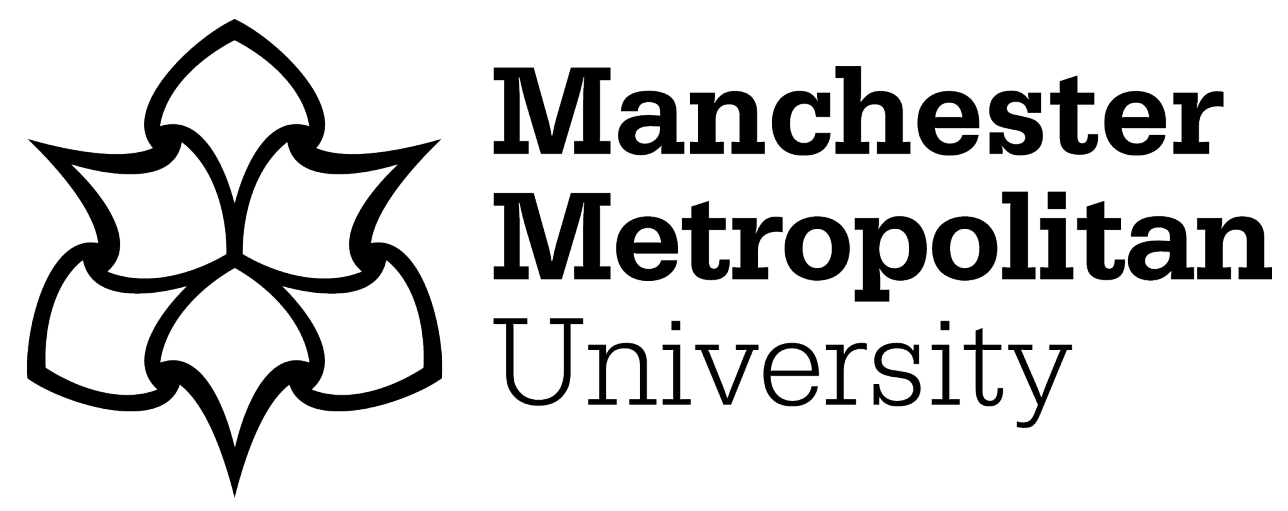

Owen, Alison L, Scholtens, Keira, Grogan, Sarah ORCID logoORCID: https://orcid.org/0000-0002-7510-765X and Burgess, Ian R (2019) Students' experiences of a facial morphing intervention designed to encourage safer drinking. Psychology and Health, 34 (8). pp. 999-1010. ISSN 0887-0446

Downloaded from: https://e-space.mmu.ac.uk/622523/

Version: Accepted Version

Publisher: Taylor \& Francis (Routledge)

DOI: https://doi.org/10.1080/08870446.2019.1584674

Please cite the published version 


\section{Students' experiences of a facial morphing intervention designed to encourage safer drinking}

Objective: The study was designed to investigate women's experiences of an age-appearance, facialageing, morphing intervention to show the effect of alcohol consumption on their skin.

Design: Seventeen women aged 19-32 took part in the study, using individual sessions where they viewed the intervention.

Main Outcome Measures: Participants were recorded during their viewing of the facial-ageing software, and the interviews recordings of the sessions were then transcribed.

Results: Transcripts were analysed using inductive thematic analysis. The women were shocked about the possible impact of alcohol consumption on their skin and appearance, and all of the women stated that the intervention had made them think about changing their alcohol consumption behaviours in the future, after viewing the images of themselves which were morphed to show the effects of both moderate and excessive alcohol consumption.

Conclusion: The results are discussed in relation to suggestions for interventions aimed at women. It is concluded that appearance-based interventions do have a role to play with regards to alcohol consumption interventions.

\section{Keywords}

Alcohol, Intervention, Women, Ageing, Appearance

\section{Introduction}


Globally, the World Health Organization (2018) reports that the harmful use of alcohol results in 3.3 million deaths each year. In England alone, medication was prescribed for the treatment of alcohol dependence in 178,247 instances, and some 200,900 hospital admissions were attributed to harmful alcohol consumption during 2011 (Health and Social Care Information Centre, 2013). Also, an estimated 595,000 adults require specialist treatment for alcohol dependency, and 167,000 working years are lost per annum due to alcohol consumption (Lawson, 2017).

Research by the National Health Service (NHS), found that binge drinking (heavy episodic drinking) in England is particularly prevalent among people aged 25 years old and under (NHS, 2011), and even more so among university students than age-matched controls in the general population (Gill, 2002). A report ordered by the House of Commons Health Select Committee (2009), found the harmful drinking habits of young people, notably university students, to be one of the main undesirable changes during the last sixty years. The Centers for Disease Control and Prevention (CDC, 2019) report that binge drinking is associated with many health problems including unintentional injuries (such as car crashes, falls, burns, and alcohol poisoning), violence (including homicide, suicide, intimate partner violence, and sexual assault) and chronic diseases (such as high blood pressure, stroke, heart disease, and liver disease).

Some attempts have already been made to reduce binge-drinking behaviours in university students. For example, eighteen recent interventions, published between 2010 and 2015, were systematically reviewed by Bridges and Sharma (2015). They found two main study designs were employed: quasiexperimental, and randomized controlled trials (RCTs), the latter design being the majority. Most of the interventions were based on brief motivational interviewing (BMI). This review concluded these interventions to be generally effective on the whole. 
Excessive alcohol consumption can have an impact on the skin and how it ages, for example in terms of causing redness to the face due to enlarged blood vessels, as well as additional impacts such as hyperpigmentation, and jaundice (Liu, Lien \& Fenske, 2010). Various studies have explored the centrality of physical appearance to female 'social currency' (Bartky, 1998; Bordo, 2003; Jackson, 1992; Jeffreys, 2005). The media places a large focus on physical appearance concerns (Grogan, 2008), for example Gallagher and Pecot-Hebert (2007) looked into the socially constructed ideals of beauty and identity represented in the media in appearance makeover television programmes, all adding to the pressure of maintaining a youthful and 'attractive' appearance.

It has been suggested that many young people feel that feel that more traditional health-related threats are too long-term to concern them or not relevant to them (Grogan \& Masterson, 2012). De Visser, Wheeler, Abraham and Smith (2013) carried out a study with 42 13-25 year olds, asking them about their thoughts and feelings about alcohol, and interventions to encourage safer drinking. One of the suggestions from participants was that they felt it would be useful to show people the impact that alcohol can have on them, rather than just telling them to avoid drinking alcohol. This suggests that an intervention where participants are provided with the evidence of what impact alcohol can have on the skin, may be effective in this age group.

Previous qualitative studies have shown personalised, age-appearance interventions to be effective in helping to show people the impact that other adverse health behaviours can have on their appearance, such as smoking (Grogan et al., 2010) and UV exposure (Williams, Grogan, Buckley \& Clark-Carter, 2012; Williams et al., 2013a; Williams et al., 2013b).

More recently, Cunningham, Stapinski, Griffiths and Baillie (2017) surveyed 138 university students in Australia, looking at dysmorphic appearance concern, alcohol use, and alcohol-related expectancies. They found a link between appearance concern and hazardous alcohol use, and that the 
link between the constructs was partially mediated by positive alcohol expectancies, suggesting that participants with appearance concerns may be more at risk for binge drinking behaviours.

Females are particularly susceptible to body dissatisfaction, and typically experience elevated levels of such appearance concerns (Grogan, 2016). Previous research with appearance-focussed facialmorphing software has indicated that men and women react differently to the software, for example Williams et al. (2013b) found that males tended to pick up more on design issues within the intervention, as well as some males being unconcerned by the facial ageing, in comparison to a similar study with women (Williams et al., 2013a). Accordingly, it was decided to focus on females only for the present study.

In 2013, the Scottish Government ran a campaign called 'Drop a glass size' using the 'Drinking Time Machine' App which used facial-ageing morphing software alongside a call to action relating to choosing smaller glasses of alcoholic drinks (Scottish Government, 2013), however there are no empirical findings about the effectiveness of the app, and what viewers thought about it. The authors could not identify any previously published work looking at the impact of appearance-focussed software for encouraging healthier alcohol consumption behaviour. Therefore, the present study used bespoke computer software to enable participants to view the impact that binge-drinking and heavy alcohol consumption may have on their appearance as they age. We considered it appropriate to carry out the present research qualitatively, to help us understand the participant's experiences as they were immersed in the intervention, and gain a full and detailed picture via analysis of the data (Smith, 2008) as no previous studies have done this in relation to alcohol.

\section{Material and Methods}

\section{Design}


The study was qualitative in design. Individual semi-structured interview sessions were used and recorded from start to finish to enable us to capture participants' initial reactions to viewing the software, and also allowed the facilitation of disclosure of sensitive information which participants may not have felt comfortable discussing in groups (Morgan, 1996).

\section{Software}

Facial-ageing morphing software was used, to enable the viewer to see how their alcohol consumption may impact on their appearance as they age. The bespoke software was created for the authors, and developed by the company 'Change my Face' (Change my Face, 2019), who design health promotion apps and software. The authors wanted to develop a piece of software that enabled the viewer to compare their faces as they would age both with the extrinsic ageing of the impact of alcohol, as well as image of their face ageing with natural, intrinsic ageing without alcohol, as opposed to just showing them the impact alcohol can have on their facial appearance without any comparison. This comparison has been used in the previous studies looking at the use of facial morphing software with alcohol and smoking, and has been found to be an effective way of showing participants the impact that external factors can have on their skin (Grogan et al., 2010; Williams et al., 2012; Williams et al., 2013a; Williams et al., 2013b).

The facilitator inputted the participants current age into the software, and the software aged the participants' photograph from their current age, to the age of 72 . The photographs showed the person's face on the left of the screen as they would age without damage from binge-drinking, and on the right of the screen as their face would age with damage from binge-drinking. This allows the viewer to compare the differences in their appearance for each age. The software was bespoke, and designed to show participants how their faces may age with the options of moderate alcohol consumption, and then excessive alcohol consumption. 


\section{Participants}

Participants were 17 female students aged between 19 and 32 years, who came from two universities in the UK. They were mainly White British (88\%) with one mixed race participant and one participant who identified as 'White not British'. All participants identified as people who drank alcohol.

\section{Materials}

Materials utilised were a laptop computer installed with the alcohol age progression software, a digital camera and an audio recorder. Additionally, an outline protocol and a list of questions were also used to ensure each session followed the same procedure. The protocol and list of questions was derived from the previous qualitative research the facilitators had carried out on smoking (Grogan et al., 2010) and UV exposure (Williams et al., 2012, 2013b).

\section{Procedure}

The intervention took place at a British University. The sessions were facilitated by research assistants who were trained in the use of the software. Potential participants were asked whether they were willing to take part in an intervention related to their alcohol consumption behaviours, and if they agreed were given the information sheet and consent form to sign.

The facilitator then took a photograph of the participant's face, and uploaded the photograph onto the age progression software. The audio recorder was then turned on, and the facilitator explained that the photographs on the left side of the screen would show their face aged if they had been drinking within the recommended guidelines, and on the right side of the screen they would see their face aged with moderate alcohol consumption, and then excessive alcohol consumption. Participants were each asked a number of questions, including, "What are your thoughts on the software?" and "Was there 
anything in particular about the pictures that made you feel like that?" At the end of the session the audio recorder was turned off.

The sessions were conducted in such a way to allow participants to discuss the issues that were important to them, and participants were encouraged to elaborate on their answers if necessary. At the end of the sessions participants were given the debrief sheet, and it was reiterated that they could contact the facilitator should they have any queries or concerns.

\section{Data Analysis}

The audio-taped individual interviews sessions were transcribed verbatim and analysed using inductive thematic analysis, by the first author, second author and research assistant. Inductive thematic analysis is a method for identifying, analysing and reporting patterns within data (Braun \& Clarke, 2006), where themes identified are strongly linked to the data themselves (Patton, 1990), and data are coded without trying to fit into a pre-existing coding frame, or the researcher's analytic preconceptions. The first and fourth author went through the transcriptions and coded the data, before the first author generated suggested themes, which were then discussed and determined by all authors. All authors agreed on the final set of themes.

\section{Results}

Four key themes arose from the thematic analysis of the interview transcripts:

\section{Impact of alcohol on appearance}

\section{Motivation to change behaviour}

\section{Effectiveness of the intervention}

\section{Suggestions for future research and practice}

Figure 1 shows the key themes and interlinking sub-themes. (Insert Figure 1 here) 


\section{Impact of alcohol on appearance}

All of the participants reported a strong immediate emotional shock reaction on viewing the photographs. This key theme incorporated two sub-themes: Shock at the impact of alcohol on facial ageing, and Difference between high consumption and moderate consumption on facial ageing.

Shock at the impact of alcohol on facial ageing

All of the participants reported an initial 'shock' reaction on viewing the photographs, and seeing the impact that alcohol could have on their facial ageing, compared to the photograph without the impact of alcohol consumption:

“Oh my god. That's awful. I think it, it's definitely shocking, erm, the difference between the photos and the skin. Just everything about it really, how the wrinkles, how your skin's gone saggy and, just in comparison with the other one, the, the colour as well. (2) Just not very nice to look at really" (Nadia)

All of the participants all pointed out specific areas of damage that particularly stood out to them in the images, in terms of what damage alcohol consumption could do to their appearance. For example, participants focussed on the colour of their skin, as well as wrinkling:

"I just look ill in general. Like pale and grey, and go them like red spots there, like the red nose, it just, the wrinkles are a lot more defined as well" (Mary)

"The forehead, yes, yes, yeah. I mean I noticed the changes mainly forehead wrinkles in the forehead, the wrinkles in the eye and the fact that the cheeks were a bit lower, the skin was a bit lower" (Ivy) 
Participants all reported that the photographs with the added impact of alcohol, were less attractive and less desirable than the photographs without alcohol consumption impact added. They also explicitly linked that it was the alcohol that was causing the damage to their skin, for example:

"Your skin just looks dead, er, not nice at all. Whereas it looks ok on the one where I just aged. People just think ooh it's just ageing, but is it? Is it showing that it's not, it's drinking and other lifestyle habits that is causing that ageing. It is really scary" (Dina)

"It's still not great even on the aged one it doesn't look that good, but on the alcohol one its awful! I've got no colouring really. And it's like the wrinkles are really deep. It's not very nice at all" (Clare)

\section{Difference between high consumption and moderate consumption on facial ageing}

The software allows users to not only compare the difference in ageing that may occur if they consume high levels of alcohol rather than moderate. Participants could really see a difference between high levels compared to moderate:

“That's so crazy. It's so much worse than the moderate one, the moderate one was bad, but that, that's so much worse. You don't think it'll have that much of an impact on you" (Mary)

"So even at moderate...that looks a hell of a lot better than high, I think I'd put myself down as quite high consumption as well" (Kate)

Participants were surprised that even at the younger ages, they were able to see a different in the skin appearance of the images, between the moderate consumption and high consumption settings, for example: 
“The 35 one too 'cause obviously, you're so young but it's still having that much of an effect on you. You still look a lot older [than the moderate consumption image]. I hope I don't look like that at 35!" (Lucy)

\section{Motivation to change behaviour}

The difference between the two photographs appeared to cause participants to feel that they were motivated to make changes to their alcohol consumption behaviour, for example:

'I've still got time to change it. It's not set in stone (.) it doesn't have to be this way (.) so it's sort of a motivational feeling really to think that I'll just make some better choices in the future I think" (Alice)

"It does actually make me think about how much I would in future from now on, just looking at the pictures of myself, obviously seeing myself, compared to seeing another person, more of a wakeup call... more alarming" (Beth)

Some participants explicitly mentioned not wanting to have a drink and it being related to what they were worried it might do to their skin, after viewing the software, for example:

"I don't want a drink....I thought that even a few drinks it can age you still, like. I was thinking I'd rather have another type of drink like a Coke or an orange juice rather than that, because your face, it just completely swells and everything. The damage you do to your skin" (Dina)

Participants did indicate that they did not feel it was too late for them to change their behaviour, and put a stop to some of the effects that alcohol might have on their skin. For example Kate said: "I think if I didn't drink as much and I ended up looking like that, at 72 I don't think I'd be too disheartened to be honest', indicating that she felt that if she changed her drinking behaviours and looked like the photograph with the intrinsic ageing alone (with no alcohol effects) then she would feel comfortable with her appearance. 
The intervention also caused a number of people to think about other lifestyle choices that they make, for example smoking.

"It's definitely given me food for thought, like it's kind of sunk in now actually, I do need to stop going out and drinking so much because it's not just that when I do go out drinking I smoke too, and that's not going to be healthy, so if you added that onto the equation then...I dread to think" (Kate)

\section{Effectiveness of the intervention}

Participants reported enjoying taking part in the intervention, and feeling that it was effective as a technique for making them consider safer alcohol consumption practices in the future.

“As an intervention...it's been quite effective on me. I know I, I don't need an intervention but it's already been quite effective on me and making me think 'uh oh maybe, maybe I shouldn't really be drinking'. I don't drink a lot but it's kind of stopping me from wanting to" (Mary)

Participants reported that they felt the intervention was effective as it was simple and easy to use.

"It looks quite straight forward. It's quite basic, it's not blinded by scientific jargon or anything" (Eleanor)

Many participants mentioned that they felt the intervention was more effective as they were seeing photographs of themselves aged, rather than just looking at a general intervention with information that wasn't about themselves.

"They have adverts and stuff and health things. But this is more personalised, because it makes it better, because seeing something saying 'oh, well that's bad for your health', it feels detached...Whereas if you use something like this, it feels very personal" (Olivia) 
Participants also reported that they felt the intervention was effective as it has demonstrated the potentially negative appearance effects of even moderate alcohol consumption, enabling a behavioural or cost/benefit judgement to be made.

"I think it helps people being more conscious and aware of what the effects is, even if here you show the pictures of moderate consumption and high consumption and there might be people that are not in this level" (Ivy)

\section{Suggestions for future practice}

Participants gave suggestions for ideas of how the research could move forward, and specific ideas for what they felt would make the intervention more effective in practice. A number of participants suggested carrying out the intervention on participants outside the age group, for example participants younger than 18 , as well as participants who are older:

"So many teenagers just go out every single weekend, they don't realise they could be doing this to themselves. Until they have the opportunity to do something like this...So maybe in schools, like high schools, and colleges, just to show them, like this could happen" (Mary)

"if you showed someone who was older then I think consumed alcohol moderately in their 40s and they showed them what they could look like if they had a high consumption of alcohol then that could definitely take an effect" (Poppy)

Another suggestion that a number of participants gave was to add health concerns to the intervention:

"I think as well as being an appearance thing for me being a health thing as well like this is what'll happen to the insides of you as well so like the effects it could have on your liver or something like that, so maybe like a double whammy, so like your appearance and your health" (Alice) 
"you could combine it with other types, 'cause it does make me feel about my, health, as well as like my appearance and you can bring more health things into that" (Olivia)

\section{Discussion}

When looking at the rates of binge drinking, and the damage this can cause to people's health, it is vitally important to develop effective interventions and strategies that have an impact on binge drinking and alcohol consumption behaviours. The results of the present study suggest that for British female university students, appearance-focussed interventions may hold some promise.

Viewing the differences in their faces when they were aged in comparison to without alcohol impacts produced the most marked impact on participants. Hevy, Pertl, Thomas, Mahler, Craig, and Ni Chuinneagain (2010) suggest that people may feel more vulnerable to developing wrinkles and age spots than to developing cancer, possibly because the former are more common and easily noticed. All of the participants reported feeling motivated to change either their alcohol consumption behaviours after viewing the photographs. This is very positive as it suggests that simply viewing the photographs may have encouraged participants to think about making changes that could benefit their health.

The findings support previous studies that have used facial-ageing software to change people's health behaviours, and add to the literature showing that appearance-focussed technology has the potential to have a really positive impact on peoples' health behaviours (Grogan et al., 2010; Williams et al., 2012; Williams et al., 2013a; Williams et al., 2013b).

\section{Methodological/Interpretative Issues}

Participants in the present study were female British students aged 19-32, all from two universities. This means that the findings need to be generalised with caution to other groups. 
It is important to consider the potential for preconceived negative stereotypes of ageing to affect people's responses. As mentioned in the introduction, there is a pressure on women to maintain a youthful appearance, for example due to media pressures (Grogan, 2008; Gallagher \& Pecot-Hebert, 2007), and therefore it may be that participants reacted to the images because of these preconceived stereotypes about ageing, and a youthful appearance. However, when analysing the transcripts, the authors did make sure to focus on instances where participants were focusing on the difference between the natural aged images and the alcohol aged images, to ensure that we were not reporting shock at simply ageing (as opposed to shock at the differences between the images).

\section{Future Research}

This study was a small-scale qualitative study, designed to assess peoples' thoughts on the intervention and look for suggestions to improve it in the future. The next step is to carry out quantitative research comparing the impact of the intervention with a health literature condition, to see if the appearance focused intervention is more effective than a health focused one. It would also be useful to carry out a qualitative study with men to see their responses to the intervention. Previous work with men with UV and smoking has suggested that men may be affected similarly to women, though it is unclear at present whether work from studies of smoking and UV will generalise to alcohol (Flett et al., 2017; Williams et al., 2013). It would also be interesting to show the software to a younger audience, to see if it would discourage them from binge drinking when they got older. Research with 11 to 14 year olds using facial morphing software to encourage safer UV exposure and sun protection behaviours found that it was effective in this age group in improving their sun protection attitudes (Owen, Grogan, Clark-Carter \& Buckley, 2018).

Additionally, existing alcohol consumption was not known for each participant before the study. It would be interesting to take this into account in future work, to gauge whether the software could be more effective for those who drink more heavily. 
Further work could utilise the intervention with also teaching participants methods for converting their good intentions to reduce alcohol consumption, into behaviour change. For example, participants could view the intervention and then take part in a session where they are taught some of the critical skills for resisting binge drinking, for example self-control and coping responses (Hendershot, Witkiewitz, George \& Marlatt, 2011).

\section{Suggestions for Practice}

As mentioned previously, the Scottish Government used an app to show people the impact of alcohol on their skin (Scottish Government, 2013). It is possible that this software could be developed into an app, which could be more widely shown to people. The software is very easy to use and follow, so it would be possible for individuals to input their own photographs, and work through the software themselves, which would be a more cost-effective method than using a face-to-face intervention.

\section{Conclusion}

Previous studies have looked at the impact of smoking and UV exposure on appearance, and peoples' responses to facial-morphing software with regards to these lifestyle habits, however this study was the first to use the facial-morphing software with regards to alcohol consumption. It has offered very promising findings, with participants reacting positively to the software, and expressing intentions to think about changing their alcohol consumption behaviours after viewing the intervention.

\section{Acknowledgements}

The authors wish to thank the participants for giving up their time to take part in the intervention, and for sharing their thoughts and feelings with us.

\section{Declaration of interest statement}

No potential conflict of interest identified. The project received no external funding.

\section{References}


Bartky SL. (1998). Foucault, femininity, and the modernization of patriarchal power. In: Weitz R, editor. The politics of women's bodies: Sexuality, appearance, and behavior. New York: Oxford University Press.

Bordo S. (2003.) Unbearable weight: Feminism, western culture, and the body. Los Angeles, CA: University of California Press. (10th anniversary ed.).

Braun, V., \& Clarke, V. (2006). Using thematic analysis in psychology. Qualitative Research in Psychology, 3, 77-101.

Change my Face (2019). Lifestyle and ageing software. Retrieved from https://changemyface.com/

Cunningham, M., Stapinski, L., Griffiths, S. \& Baillie, A. (2017). Dysmorphic Appearance Concern and Hazardous Alcohol Use in University Students: The Mediating Role of Aleohol Expectancies. Australian Psychologist, 6, 424-432.

de Visser, R.O., Wheeler, Z., Abraham, C. \& Smith, J.A. (2013) 'Drinking is our modern way of bonding': Young people's beliefs about interventions to encourage moderate drinking. Psychology \& Health, 28, 1460-1480.

Flett, K., Grogan, S., Clark-Carter, D., Gough, B \& Conner, M. (2017). Male smokers' experiences of an appearance-focused facial-ageing intervention. Journal of Health Psychology, 22, 4, 422-433.

Gallagher, A.H. \& Pecot-Hebert, L. (2007) "You Need a Makeover!": The Social Construction of Female Body Image in "A Makeover Story", W "hat Not to Wear", and "Extreme Makeover", Popular Communication, 5:1, 57-79,

Grogan, S. (2016). Body image: Understanding body dissatisfaction in men, women and children (3 ${ }^{\text {rd }}$ ed). London: Routledge. 
Grogan, S., Flett, K., Clark-Carter, D., Gough, B., Davey, R., Richardson, D., \& Rajaratnam, G. (2010). Women Smokers' Experiences of an Age-Appearance Anti-Smoking Intervention: A Qualitative Study. British Journal of Health Psychology, 16, 675-689.

Grogan, S. \& Masterson, D. (2012). Using Appearance Concerns to Promote Health. In N. Rumsey \& D. Harcourt (eds) pp. 594. The Oxford Handbook of The Psychology of Appearance. Oxford: Oxford University Press.

Health and Social Care Information Centre (2013). Statistics on Alcohol - England.

Hendershot, C.S., Witkiewitz, K., George, W.H. \& Marlatt, G.A. (2011). Relapse prevention for addictive behaviours. Substance Abuse Treatment, Prevention, and Policy, 16, 17.

House of Commons Health Committee (2009). Alcohol. Retrieved from http://www.publications.parliament.uk/pa/cm200910/cmselect/cmhealth/151/15102.htm

Jackson, L. A. (1992). Physical appearance and gender: Sociobiological and sociocultural perspectives. Albany, NY: SUNY Press.

Jeffreys S. (2005). Beauty and misogyny: Harmful cultural practices in the West. New York: Routledge.

Lawson, M. (2017). Alcohol: The Facts. Retrieved from https://www.alcoholconcern.org.uk/Blog/alcohol-the-facts

Liu, S.W., Lien, M.H. \& Fenske, N.A. (2010). The effects of alcohol and drug abuse on the skin. Clinics in Dermatology, 28, 391-399.

Morgan, D. L. (1996). Focus groups. Annual Review of Sociology, 22, 129-152. 
Williams, A.L., Grogan, S., Buckley, E. \& Clark-Carter, D. (2012). A qualitative study examining women's experiences of an appearance-focussed facial-ageing sun protection intervention. Body Image, 9, 417-420.

Williams, A.L., Grogan, S., Buckley, E. \& Clark-Carter, D. (2013a). British adolescents' experiences of an appearance-focussed facial-ageing sun protection intervention: a qualitative study. Education and Health, 31, 97-101.

Williams, A.L., Grogan, S., Buckley, E. \& Clark-Carter, D. (2013b). Men's experiences of an appearance-focussed facial-ageing sun protection intervention: A qualitative study. Body Image, 10, 263-266.

World Health Organization (2018). Management of substance abuse. Retrieved from http://www.who.int/substance abuse/facts/en/ 


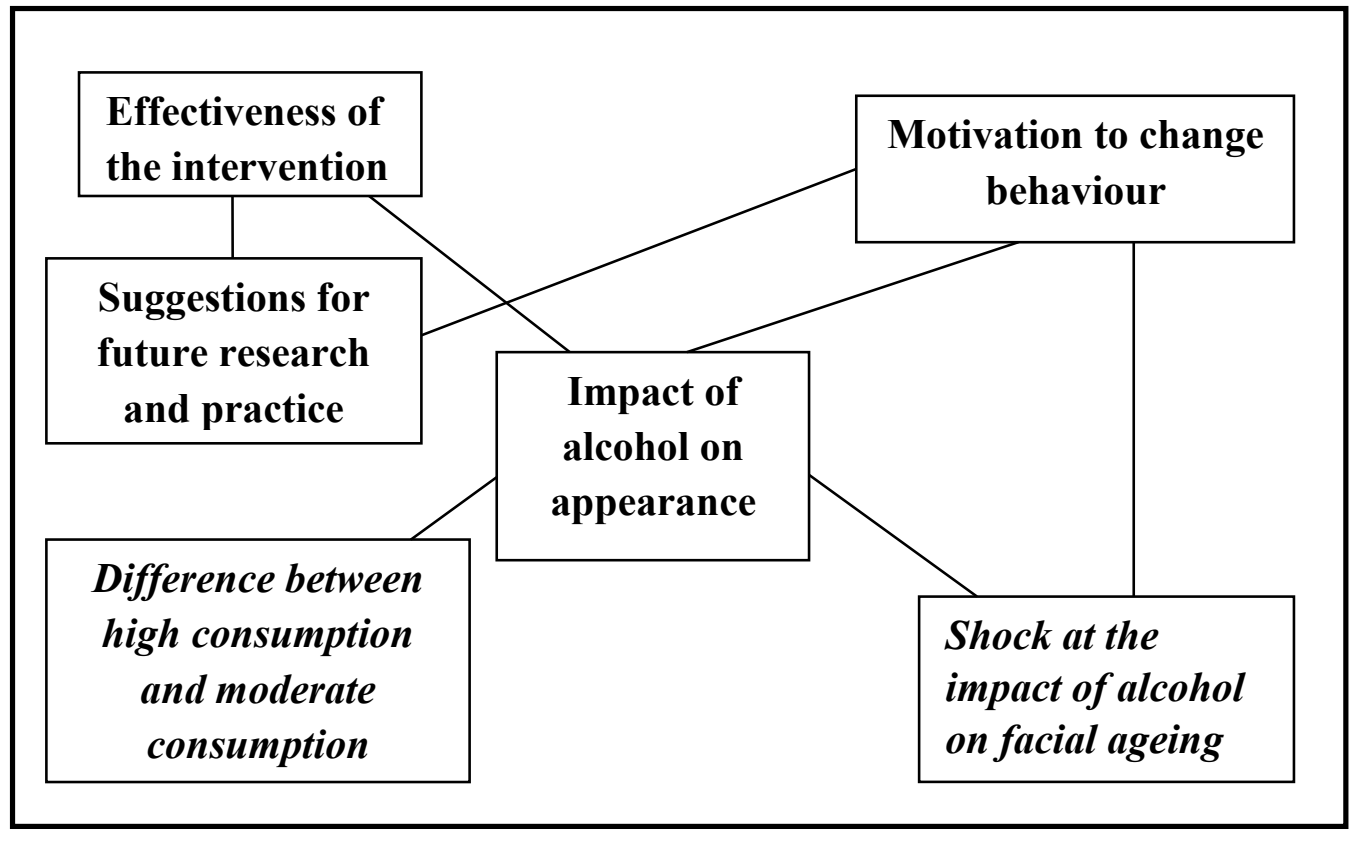

Figure 1. The key themes that arose from the individual sessions, and the interlinking sub-themes 